\section{$\underset{\substack{\text { hommes } \\ \text { \& migrations }}}{ }$}

\section{Hommes \& migrations}

Revue française de référence sur les dynamiques

migratoires

1316 | 2017

L'islam en Europe

\title{
Un paradoxe belge
}

Quarante ans de reconnaissance et d'altérisation de l'islam en Belgique

\section{Hanifa Touag}

\section{Q OpenEdition \\ 1 Journals}

\section{Édition électronique}

URL : http://journals.openedition.org/hommesmigrations/3793

DOI : 10.4000/hommesmigrations.3793

ISSN : 2262-3353

\section{Éditeur}

Musée national de l'histoire de l'immigration

\section{Édition imprimée}

Date de publication : 1 mars 2017

Pagination : 49-56

ISBN : 978-2-919040-37-7

ISSN : $1142-852 X$

\section{Référence électronique}

Hanifa Touag, « Un paradoxe belge », Hommes \& migrations [En ligne], 1316 | 2017, mis en ligne le 01 mars 2020, consulté le 18 septembre 2020. URL : http://journals.openedition.org/hommesmigrations/ 3793 ; DOI : https://doi.org/10.4000/hommesmigrations.3793 


\title{
UN PARADOXE BELGE : QUARANTE ANS DE RECONNAISSANCE ET D'ALTÉRISATION DE L'ISLAM EN BELGIQUE
}

Par HANIFA TOUAG, doctorante en sociologie à l'Institut d'études politiques de Paris et à l'Université catholique de Louvain.

\author{
La montée récente du «problème musulman » dans les débats \\ publics en Belgique renseigne sur la situation paradoxale \\ qu'occupe l'islam dans le pays. À la différence de son voisin \\ français, la religion musulmane a bénéficié d'un long parcours \\ de reconnaissance de la part des autorités belges. Pour autant, \\ cette religion n'échappe pas aux stigmatisations dont ses \\ pratiquants sont victimes partout dans une Europe gagnée \\ par le néolibéralisme. Sommés de s'intégrer dans une société \\ civile tâchant de gérer ses identités multiples, ils sont soumis \\ à des processus idéologiques qui jouent de l'amalgame avec \\ les figures radicalisées de leur religion et produisent leur \\ altérisation.
}

Les attentats de Paris et de Saint-Denis puis ceux de Bruxelles et de Zaventem au cours de l'année 2015-2016 ont suscité un émoi considérable au sein de la société belge, pas seulement parce que ces attaques frappent désormais la Belgique, mais aussi et surtout parce que des Belges y ont pris part $^{1}$. Dès lors, se sont multipliées les manifestations identitaires hostiles à l'islam, se traduisant notamment par des agressions à caractère islamophobe telles des dégradations de mosquées ou une marche de skinheads d'extrême droite quelques jours après les attentats de Bruxelles² ${ }^{2}$ Ces événements s'inscrivent dans un contexte politique déjà très sensible du fait de la distension croissante du lien social et national entre Wallons et Flamands, au point de menacer l'unité et le vivre ensemble de l’État fédéral.

Ce problème n'est cependant pas nouveau puisque des recherches ont signalé, avant les attentats, une tendance à la polarisation croissante de la société belge dans son rapport à l'islam. Une enquête menée il y a deux ans par le Cismoc de l'université catholique de Louvain a ainsi mis en évidence que la question de l'islam cristallise un grand nombre de peurs et d'angoisses en Belgique, au risque de favoriser l'idée d'un islam monolithique 
et, par conséquent, de l'essentialiser ${ }^{3}$. De tels fantasmes contribuent à occulter la diversité de l'islam tant sur le plan de ses courants que des modes de croire propres à chaque individu, la présence historique de l'islam en Belgique bien avant les migrations économiques des années 1960 et les multiples efforts déployés à la fois par des musulmans et des non-musulmans pour tisser des liens entre les communautés et favoriser la coexistence religieuse. Si cette situation est comparable à celle de ses voisins européens, en La Belgique faitfigure premier lieu la France et le de véritable avant-garde,

car elle est le premier

pays à avoir procédé

à une politique active de reconnaissance caractérisée par des relations plus étroites et régulières avec chaque culte, tout en préservant le devoir de neutralité. Royaume-Uni au sein desquels se trouvent d'importantes minorités musulmanes, elle apparaît toutefois difficilement compréhensible au regard de l'absence d'un passé colonial belge dans les pays musulmans.

Cette situation est d'autant encore la question de son intégration ? L'idée selon laquelle l'institutionnalisation de l'islam aboutirait mécaniquement à sa normalisation ne cesse de se renforcer, d'autant plus qu'elle fait écho à un dysfonctionnement réel des institutions représentatives de l'islam. Toutefois, cette idée ne prend pas en compte la dimension plurifactorielle dudit " problème musulman » qui relève surtout d'une construction et de projections d'acteurs, de réseaux et d'institutions que la problématisation de l'islam sert et conforte dans des sphères distinctes (politique, médiatique et religieuse). plus surprenante que la Belgique a très rapidement entrepris une reconnaissance institutionnelle de l'islam, conférant ainsi de nombreux droits aux musulmans au même titre que pour l'ensemble des cultes déjà reconnus. En ce sens, la Belgique fait figure de véritable avantgarde, car elle est le premier pays à avoir procédé à une politique active de reconnaissance caractérisée par des relations plus étroites et régulières avec chaque culte, tout en préservant le devoir de neutralité. Quarante ans plus tard, le bilan apparaît très mitigé pour un certain nombre d'observateurs de plus en plus tentés de remettre en cause la politique du multiculturalisme confessionnel qui prévalait jusqu'alors ${ }^{4}$.

Cette contribution s'intéresse à la situation paradoxale de l'islam en Belgique : alors que l'islam bénéficie de droits culturels et cultuels importants au titre de religion officiellement reconnue depuis 1974, comment expliquer que se pose

\section{La reconnaissance problématique de l'islam en Belgique}

Fruit d'un compromis acté en 1831 entre le jeune État belge et l'Église catholique, le dispositif de reconnaissance permet en théorie à tout temporel du culte reconnu (désormais au nombre de sept : le catholicisme, le protestantisme, l'anglicanisme, le judaïsme, l'islam, la religion orthodoxe et le bouddhisme) de jouir d'un certain nombre d'avantages matériels et symboliques tels que la prise en charge des traitements et des pensions des ministres du culte et des aumôniers pénitentiaires, le financement du déficit des établissements publics et, depuis le Pacte scolaire (1959), l'organisation de cours de religion dans les écoles officielles ${ }^{5}$. Nous ne reviendrons pas en détail sur les différentes étapes de ce processus pour nous concentrer sur la différence entre les principes régissant ce dispositif et leur application s'agissant du culte islamique.

Le processus ayant conduit à la reconnaissance officielle du culte islamique est amorcé à la fin des années 1960. À cette date, les populations musulmanes issues de l'immigration (essentiellement marocaine et turque $)^{6}$ sont peu structurées et restent à la marge des décisions qui les 
concernent ${ }^{7}$. Néanmoins, les difficultés éprouvées par les travailleurs musulmans pour pratiquer leur religion dans des conditions décentes amènent le législateur belge, d'une part, à émettre au cours de l'année 1970 une proposition parlementaire, prélude à la reconnaissance de l'islam ${ }^{8}$, d'autre part, à inscrire cette démarche dans le cadre d'une politique plus générale d'accompagnement et d'intégration des populations étrangères. Lorsque le temporel du culte islamique est officiellement reconnu quatre ans plus tard, le Centre islamique et culturel (CIC), association née à la fin des 1950 et regroupant à l'origine des étudiants arabes, des réfugiés albanais et quelques convertis belges, est désigné comme le principal interlocuteur de l'État. Bien que peu représentatif des populations musulmanes d'origine marocaine et turque, le CIC est perçu comme un partenaire rassurant pour l'État belge, et ce en raison du soutien dont le centre bénéficie alors de la part des pays musulmans, au premier rang desquels se trouve l'Arabie saoudite. Lionel Panafit rappelle que la désignation du CIC comme interlocuteur provisoire répond à des considérations d'ordres économique et politique ${ }^{9}$, et ne tient pas compte des orientations religieuses et doctrinales wahhabites de l'État saoudien avec lequel des entreprises belges négocient dans la même période des contrats en vue de la construction de vastes complexes hospitaliers. Pour autant, le CIC, qui conserve une relative autonomie vis-àvis de l'Arabie saoudite jusqu'à son rattachement à la Ligue islamique mondiale en 1982, contribue à la mise en œuvre de la reconnaissance de l'islam à travers la désignation des professeurs de religion islamique. Ainsi, entre 1975 et 1989, le CIC se chargea de désigner des professeurs de religion islamique, jouant ainsi un "rôle d'organe de chef de culte informel $l^{10} »$.

\section{Une application tardive et incomplète génératrice de frustrations et d'inégalités}

L'écart entre les objectifs visés et leur application n'a cependant cessé de se creuser, si bien qu'il a fallu plus de trente ans pour que les premières mosquées soient reconnues. Ce décalage s'explique principalement par l'instabilité même du processus de reconnaissance, liée non seulement à la succession d'évènements locaux et internationaux impliquant l'islam,

mais également à la réaction des acteurs politiques et religieux dans un climat devenu progressivement délétère.

La décennie 1980 est, de ce point de vue, emblématique : alors qu'émerge une conception militante et politique de l'islam à l'échelle internationale ${ }^{11}$ Lorsque le temporel du culte islamique est officiellement reconnu quatre ans plus tard, le Centre islamique et culturel (CIC), association née à la fin des 1950 et regroupant à l'origine des étudiants arabes, des réfugiés albanais et quelques convertis belges, est désigné comme le principal interlocuteur de l'État. parallèlement à la montée des revendications de type identitaire à l'échelle, cette fois, nationale ${ }^{12}$, l'État belge soumet la formation du futur organe représentatif du culte islamique à un ensemble de critères de plus en plus précis (le nombre de femmes, l'égale proportion de néerlandophones et de francophones,

5. Signé en 1958, le Pacte scolaire contraint les écoles officielles à organiser des cours de religion correspondants aux différents cultes reconnus, ainsi qu'un cours de morale non confessionnelle. 6. Ces migrations économiques s'inscrivent dans le cadre des accords bilatéraux signés en 1964 entre la Belgique, la Turquie et le Maroc. 7. Hassan Bousetta, Brigitte Maréchal, L'islam et les musulmans en Belgique : enjeux locaux et cadres de réflexion globaux, Bruxelles, Fondation Roi Baudouin, 2003. 8. Jean-François Husson, «La reconnaissance et le financement public de l'islam en Belgique : entre liberté de religion et dimension sécuritaire ", Note de l'Observatoire politique du religieux, 2016. 9. Lionel Panafit, « Les problématiques de l'institutionnalisation de l'islam en Belgique (1965-1996)", in Felice Dassetto (dir.), Facettes de l'islam belge, Louvain-la-Neuve, Academia-Bruylant, 1997. 10. Caroline Sägesser, Corinne Torrekens, "La représentation de l'islam ", in Courrier hebdomadaire du CRISP, $n^{\circ}$ 1996-1997, 2008, pp. 5-55. 11. L'arrivée au pouvoir de l'ayatollah Khomeini en Iran en 1979, sa fatwa condamnant l'auteur des Versets Sataniques en 1988 suivi de l'assassinat de l'imam-directeur de la Grande Mosquée de Bruxelles en février 1989. 12. Une manifestation est organisée à Bruxelles en 1986 au moment l'intervention américaine en Libye et l'islam y est mobilisé. Voir Felice Dassetto et Albert Bastenier, Medias u akbar. Confrontations autour d'une manifestation, Louvain-la-Neuve, Ciaco, 1987. L'année 1989 a été celle du déclenchement, en octobre, de l'affaire du "voile islamique » dans une école secondaire de Molembeek et celle de l'ouverture de la première école libre islamique (école al-Ghazali) un mois plus tôt. 
la représentativité, la modération...) qu'aucun autre culte reconnu n'est tenu d'observer. L'organisation des premières élections visant à doter le temporel du culte d'un organe représentatif est révélatrice des contradictions induites par le croisement de ces critères. En septembre 1990, l'État décide d'invalider le processus électoral mis en place quelques mois plus tôt par le Centre islamique et culturel, et parti-

En septembre 1990, l'État décide d'invalider le processus

électoral mis en place quelques mois plus tôt par le Centre islamique et culturel, et participe à la création du

Conseil provisoire des sages, composé en partie de membres nommés par le gouvernement.

cipe à la création du Conseil provisoire des sages, composé en partie de membres nommés par le gouvernement. Le revirement de l'État dans ses relations avec le CIC part d'un double constat : à la fois celui d'un manque de représentativité du CIC par rapport aux communautés musulmanes dont il n'est pas issu et celui de propos de son directeur jugés controversés dans un contexte de sensibilité politique accrue sur la question du radicalisme musulman. Mais l'intervention de l'État pour disposer d'un interlocuteur modéré et représentatif apparaît comme une tentative d'ingérence contradictoire avec son devoir de neutralité et est, par conséquent, illégitime aux yeux de la communauté musulmane. Les musulmans se retrouvent ainsi dans une situation de double bind (" injonction contradictoire »), c'est-à-dire sommés de choisir leurs représentants de manière démocratique mais tout en étant conformes aux critères de modération fixés par l'État, quitte à valoriser des individus davantage issus des milieux sécularisés (partis, syndicats...) que religieux. Désavoué par les mosquées qui ne lui reconnaissent aucune compétence théologique, le Conseil provisoire des sages périclite et cesse ses activités en $1992^{13}$. Après une période de blocage, le processus de reconnaissance du culte musulman a repris dans un contexte plus favorable à l'apaisement des relations avec l'islam. En mars 1997, la décou- verte du corps de Loubna Benaïssa, fillette assassinée cinq ans plus tôt, suscite un émoi considérable dans l'opinion publique belge et entraîne un mouvement de solidarité plus général envers la communauté musulmane. Dans ces circonstances, l'Exécutif provisoire des musulmans, chargé de préparer l'avènement d'une instance représentative stable - l'Exécutif des musulmans de Belgique -, élabore une série de propositions pour l'organisation du scrutin dont certaines seront retenues dans le cadre des élections du 13 décembre 1998. Ainsi, et suivant les recommandations de l'Exécutif provisoire, une assemblée de 68 membres, comprenant 51 membres élus dans les mosquées et dans d'autres lieux publics et 17 membres cooptés, est installée le 22 janvier 1999. Quelques jours plus tard, elle remet au ministre de la Justice une liste de dixsept candidats à l'Exécutif. Cette dernière est l'objet d'un contrôle de sécurité (screening) de la part de la Sûreté de l'État qui décide d'écarter l'un des candidats choisis par peur de voir ressurgir la menace du GIA ${ }^{14}$. Mais cette approche sécuritaire fournit dès lors le prétexte à une lutte de pouvoirs interne entre l'Assemblée et l'Exécutif.

\section{La représentation de l'islam en Belgique, enjeu d'une lutte de pouvoir}

En janvier 2001, l'Assemblée émet un vote de défiance à l'égard de l'Exécutif qu'elle juge dépourvu de légitimité démocratique et religieuse. Mobilisé pour concurrencer l'Exécutif, l'argument du « déficit démocratique et religieux " sert en partie des acteurs représentant les intérêts des pays d'origine (le Maroc et la Turquie) et espérant jouer un rôle prépondérant dans les instances de représentation de l'islam en Belgique. L'année 2001 marque ainsi le début d'une longue période de crise de la représentation de l'islam en Belgique, alimentée par les 
tensions internes décrites précédemment, puis aggravée par l'intervention des pouvoirs publics ainsi que par les soupçons de détournements de fonds publics visant l'Exécutif en 2005. La crise culmine en 2007 et débouche sur la paralysie totale de l'institution, que l'État cherche à résoudre; mais son action tend à renforcer le cercle vicieux de l'ingérence étatique - par le biais du ministère de la Justice - en partie responsable des difficultés puisque cette intervention attise des tensions déjà vives. Il s'ensuit une polarisation entre les partisans et les opposants à la médiation de l'État, les seconds optant alors pour une stratégie de réaction préjudiciable pour l'image de l'Exécutif des musulmans : ainsi, ce dernier s'est-il enfermé dans le mutisme suite à l'assassinat du réalisateur Theo Van Gogh en novembre 2004. Si l'Exécutif des musulmans semble avoir trouvé une récente stabilité, il n'en demeure pas moins la cible de diverses tentatives d'ingérence de la part des représentants des pays d'origine des nombreuses communautés musulmanes de Belgique. Plus encore, malgré l'avancée de certains sujets tel celui du recrutement des professeurs de religion islamique - désormais au nombre de 70 -, de nombreux dossiers ont été laissés en suspens comme la formation des professeurs de religion islamique et des ministres du culte, la reconnaissance et le financement des mosquées ou encore la définition des programmes de religion islamique pour les classes du secondaire.

Ce statu quo qui a perduré pendant 40 ans a eu d'importants effets identitaires et symboliques au sein des communautés musulmanes : il a, en effet, alimenté un terrain propice à la diffusion de représentations stéréotypées de l'islam et leur intériorisation progressive par les nouvelles générations de croyants. De surcroit, cette situation a favorisé le recours constant à des imams de pays d'origine comme le Maroc, et même à de jeunes imams formés au wahhabisme

saoudien. Or ce personnel religieux se trouve à contre-courant des mours et de la culture politique et sociale de la Belgique, suscitant dès lors un tiraillement croissant des fidèles entre une norme "musulmane " présentée comme absolue et intangible et des normes sociales exigeant une adaptation du discours et des pratiques religieuses : ainsi, la théorie évolutionniste de Darwin ou encore l'égalité des sexes sont contestées par les tenants d'une vision littéraliste et traditionaliste de l'islam ${ }^{15}$.

De plus, le statu quo a pénalisé les ressources financières du culte musulman, ce dernier ne bénéficiant pas des mêmes avantages que les autres cultes alors même que la demande religieuse musulmane est proportionnellement plus importante. La chercheuse Corinne Torrekens met en évidence à l'échelle des communes des différences de traitement significatives entre les différents cultes reconnus, au détriment de l'islam ${ }^{16}$. Une telle situation renforce l'impression que l'islam constitue une religion de seconde zone, nourrissant en retour le sentiment d'injustice d'une partie des musulmans ${ }^{17}$.

Le problème de l'intégration de l'islam ne peut être saisi qu'à la lumière du dysfonctionnement réel

Si l'Exécutif des musulmans semble avoir trouvé une récente stabilité, il n'en demeure pas moins la cible de diverses tentatives d'ingérence de la part des représentants des pays d'origine des nombreuses communautés musulmanes de Belgique.

des instances représentatives des musulmans de Belgique. En l'absence d'une hiérarchie clairement établie - et a fortiori au sein du sunnisme qui regroupe la grande majorité des musulmans de Belgique -, ont lieu d'incessantes divisions et luttes pour exercer le leadership au sein de la communauté. Mais réduire ce problème à la seule question institutionnelle revient à imputer la responsabilité des difficultés à un seul acteur, en l'occurrence la communauté musulmane 
jugée incapable de s'organiser par elle-même, et à ignorer un contexte faisant perdurer les dysfonctionnements précédemment évoqués. De fait, constituant initialement une "structure structurée " - c'est-à-dire un symptôme -, ces derniers sont progressivement devenus une "structure structurante ", autrement dit l'une des causes - sans être la seule et unique - du problème de l'intégration de l'islam.

\section{L'islam et l'identité pris au piège du néolibéralisme}

Le problème de l'intégration de l'islam prend également sa source dans les représentations d'acteurs, de groupes et d'institutions qui œuvrent séparément à l'altérisation de l'islam, c'est-àdire à sa construction comme une réalité radicalement étrangère au contexte belge, mais qui ont pour point commun d'adhé-

Le problème de l'intégration de l'islam prend également sa source dans les représentations d'acteurs, de groupes et d'institutions qui œuvrent séparément à l'altérisation de l'islam, c'est-àdire à sa construction comme une réalité radicalement étrangère au contexte belge, mais qui ont pour point commun d'adhérer aux valeurs et aux principes du néolibéralisme. de réglementation à tous les niveaux, ce qui revient notamment à remettre en cause toute forme d'intervention de l'État dans la sphère économique et les diverses institutions régissant la vie politique et sociale, aussi bien les partis que les instances religieuses par exemple. Cette idéologie cherche dès lors à peser dans l'actuelle mondialisation pour constituer un cadre politique, économique et socioculturel apte à satisfaire ses objectifs. En ce sens, elle contribue à l'apparition d'individus isolés et atomisés, désireux de se positionner comme des individus singuliers et pour lesquels la moindre réglementation apparaît insupportable et illégitime.

En Belgique, le néolibéralisme s'est précocement institué du fait de la pression du mouvement flamand pour attirer davantage les grandes firmes multinationales (FMN) en Flandre - et ainsi devenir le nouveau pôle tertiaire dynamique du pays au détriment de la Wallonie industrielle -, avant de se généraliser progressivement à l'ensemble des politiques nationales à partir des années 1980 sous la pression des institutions européennes ayant opté pour une telle orientation afin de demeurer compétitives au niveau international ${ }^{18}$. Cette nouvelle donne s'est d'abord répercutée au niveau des structures médiatiques et politiques du pays. Dans le premier cas, se sont opérées une concentration progressive des médias et une disparition concomitante des canaux alternatifs incapables de faire face aux nouvelles exigences de rentabilité et d'instantanéité, qui ont poussé les organes traditionnels (télévision, radio, presse écrite) à opter pour une stratégie de surenchère ${ }^{19}$. L'essor d'Internet a renforcé ce mouvement en dérégulant davantage la sphère médiatique traditionnelle désormais touchée par une défiance accrue des citoyens à leur égard du fait de la multiplication des sources d'information.

De leur côté, la majorité des partis politiques ont progressivement abandonné le projet de transformation des conditions socioéconomiques au profit d'un « consensus mou » caractérisé par une entente cordiale avec les partis flamands et un investissement prioritaire en faveur de la construction européenne. Ce changement d'orientation politique n'a pu empêcher l'affirmation de mouvements et de partis nationalistes ou séparatistes en Flandre et plus rarement en Wallonie, dont la tradition xéno- 
phobe a petit à petit pris la forme d'un discours stigmatisant l'islam et les musulmans ${ }^{20}$. Confrontés à l'érosion de leur électorat tenté par une plus grande fermeté à légard des musulmans, certains hommes politiques issus de la droite libérale ont fait de la neutralité un sujet politique central en devenant ses principaux défenseurs, leur permettant de dénoncer une gauche socialiste accusée de naïveté sur la question de l'islam.

\section{L'altérisation de l'islam dans le débat public belge}

C'est dans ce contexte que l'islam a pris une place sans cesse croissante dans les débats publics. Opérant désormais dans un cadre hyperconcurrentiel qu'ils ont accepté, les médias ont contribué à la construction et à la diffusion des stéréotypes sur l'islam et les musulmans, à l'image de ce qui s'est produit en France ${ }^{21}$. Ainsi, la publication de L'Iris et le croissant par Felice Dassetto en 2013 a été l'occasion d'un emballement médiatique sans précédent, qui s'est notamment traduit par la focalisation sur le fantasme d'un " grand remplacement " démographique à Bruxelles. De même, le magazine Le Vif-L'Express multiplie les couvertures consacrées à l'islam politique au détriment des autres formes d'islam dépourvues de projet politique ${ }^{22}$.

Les partis politiques ont également joué un rôle essentiel dans l'édification d'un " problème musulman », à commencer par le parti d'extrême droite Vlaams Block - ex Vlaams Belang - pour lequel l'islam prend une place importante comme l'attestent les propos de son leader Filip Dewinter. En effet, l'islam a permis à ce parti de capitaliser sur un thème moins polémique que l'éclatement de la Belgique auquel appelle le Vlaams
Block. En s'appropriant la même thématique, le parti d'obédience ultra-nationaliste NVA de Bart de Wever a obtenu l'élection de 27 députés lors des législatives de 2010. Du côté wallon, l'extrême droite compense sa faiblesse électorale en exerçant son influence sur Internet : des entrepreneurs d'identité relaient et banalisent les thèses xénophobes, assimilant à excès islam, étrangers, délinquance et terrorisme. C'est ainsi que les sites « L'Union-faitla-force.be » et "Nonali. Les partis politiques ont be " se consacrent entièrement à évoquer la question de l'islamisme tout en spéculant sur un complot véhiculé par les partis politiques traditionnels de concert avec les immigrés.

Tous ces bouleversements participent à l'« altérisation " progressive de également joué un rôle essentiel dans l'édification d'un «problème musulman ") à commencer par le parti d'extrême droite Vlaams Block - ex Vlaams Belang - pour lequel I'islam prend une place importante comme l'attestent les propos de son leader Filip Dewinter.

\section{l'islam. Inspiré des travaux} de Gayatri Spivak ${ }^{23}$ sur les identités postcoloniales, le concept d'altérisation - « othering »identifie deux groupes : un dominant qui altérise et un dominé qui subit. Cependant, cette définition néglige le fait que le dominé ne se contente pas de subir, mais au contraire participe au processus même d'altérisation, et ce d'autant plus que le néolibéralisme encourage l'expression d'une identité singulière pour chaque individu indépendamment des institutions traditionnelles. L'islam leur offre alors l'opportunité de s'affirmer plus facilement en comparaison d'autres éléments constitutifs de l'identité nécessitant davantage de ressources et d'implication comme l'identité professionnelle, politique ou encore culturelle. En Belgique, depuis les années 1990, ces individus convergent dans des groupes à tendance littéra-

21. Thomas Deltombe, L'islam imaginaire. La construction médiatique de l'islamophobie en France, 1975-2005, Paris, La Découverte, 2005. 22. En témoignent plusieurs unes du magazine dont les titres, se référant de façon quasi-explicite aux thèses du grand remplacement, sont particulièrement anxiogènes : " Bruxelles, musulmane en 2030 ? " (16/04/2010); "Mangez-vous halal sans le savoir?" (28/05/2010) ; " Partis politiques, dévoilez-vous ! " (18/og/2009); " Comment l'islam menace l'école " (29/08/2008) ; "Les convertis. Ces Belges, fous d'Allah " (7/09/2007) ; "Peut-on encore critiquer l'islam ? " "Comment les Frères musulmans ont pris en otage la Belgique " (6/03/2015), etc. 23. Gayatri Chakravorty Spivak, "Can the Subaltern Speak? ", in Cary Nelson, Lawrence Grossberg (dir.), Marxism and the Interpretation of Culture, Chicago, University of Illinois Press, 1988. 
liste, notamment tabligh et salafiste, ou politique comme le Parti citoyenneté et prospérité (PCP) ou plus récemment le Parti Islam.

À l'issue de l'examen des différents acteurs impliqués dans l'altérisation de l'islam, il apparait que le multiculturalisme confessionnel à la belge ne peut constituer une solution pleinement satisfaisante au problème de "l'intégration de l'islam ». En effet, la reconnaissance de l'islam se heurte à la dérégulation de l'information et ne peut s'opposer à l'importation de problématiques internationales via les réseaux sociaux et Internet : la mémoire de Mohamed Merah, l'auteur des attentats ayant frappé la France en mars 2012, a été entretenue par le groupe Sharia 4 Belgium, rendant impossible d'isoler la question de l'islam en Belgique de l'influence de ses voisins européens. Par ailleurs, la reconnaissance de l'islam s'inscrit dans un cadre exclusivement communautaire, sans prendre en compte l'action des agents non musulmans et leur rôle prééminent dans la diffusion de l'idée selon laquelle l'islam est un phénomène par essence étranger aux mœurs et aux modes de vie belges. La volonté d'investir la reconnaissance de l'islam d'une mission intégratrice sur-responsabilise les personnels du culte (imams, professeurs de religion islamique) qui éprouvent un profond malaise lorsque l'islam est mobilisé au moment d'un attentat, et ce tout en dédouanant les entrepreneurs de l'altérisation.

Le paradoxe d'une altérisation parallèle à un processus de reconnaissance de l'islam entamé dans les années 1970 s'explique donc par les limites intrinsèques du multiculturalisme confessionnel dans un cadre néolibéral, attisant en Belgique comme ailleurs en Europe des tensions identitaires qui franchissent les frontières nationales et se répercutent dans une vie politique, médiatique et sociale reconfigurée depuis plus de trente-cinq ans. La question de l'intégration de l'islam ne peut donc se réduire à son seul versant religieux, mais renvoie à un problème plus large impliquant tous les acteurs de la sphère publique. 